\title{
An efficient Monte Carlo Potts method for the grain growth simulation of single-phase systems
}

\author{
Noureddine Maazi* (iD, Balahouane Lezzar \\ Laboratoire Microstructures et défauts dans les Matériaux (LMDM), Département de Physique, Faculté des Sciences Exactes, \\ Université Mentouri Constantine 1, Algeria.
}

\begin{abstract}
The choice of the lattice sites to be reoriented in the Monte Carlo Potts algorithm for grain growth simulation is repeated in a non-homogeneous way. Therefore, some grains are favorably growing than others. This fact may seriously affect the simulation results. So a modified MC method is presented. Lattice sites are selected for reorientation one by one following their positions in the matrix in each Monte Carlo step (mcs). This approach ensures that the various selections of one lattice site within every mcs are eliminated, and no favorable growth of grains at the expense of others. The calculation time considerably decreases. The effect of real-time and physical temperature on the grain growth kinetics is discussed.
\end{abstract}

Keywords: alloys, crystal growth, Monte Carlo simulation, microstructure.

\section{Introduction}

The microstructure of polycrystalline materials is an important factor that controls their physical and mechanical properties. The process of grain growth is driven by the reduction of grain-boundary (GB) area, i.e., minimization of GB energy. The larger grains grow at the expense of the smaller neighboring ones. As a result of small grains vanishing, the average grain size $\bar{R}$ increases (Atkinson, 1988). Grain growth in polycrystalline materials has been investigated with many computer simulation techniques that include Monte Carlo Potts (MC) (Anderson et al., 1984; Srolovitz et al., 1986; Holm et al., 1991), vertex (Kawasaki et al., 1989; Weygand et al., 2000), cellular automata (Geiger et al., 2001; Raghavan \& Sahay, 2007), and phase-field (Suwa et al., 2006; Wang, 2006). Because of its simplicity, the MC method has been extensively used to simulate several aspects of grain growth kinetics. At a mesoscopic scale, the MC method treats the evolution of the non-equilibrium discrete ensemble, which represents the microstructure. Although this technique has presented successful results in simulating grain growth behavior such as grain growth kinetics and pinning effect by second phase particles (Messina et al., 2001; Kim, 2010; Phaneesh et al., 2012), this method requires a long calculation time due to its probabilistic nature. To reduce the simulation runtime, some modifications have been proposed to the basic MC algorithm (Radhakrishnan \& Zacharia, 1995; Holm \& Battaile, 2001; Yu \& Esche, 2003a). In the basic MC algorithm, the probability for possible site selection is not equal for all lattice sites, i.e., the reorientation test of lattice sites is repeated in a non-homogeneous way. Therefore some grains are favorably growing than others.

*Corresponding author: nmaazi@yahoo.fr

ORCID ID: 0000-0001-8364-6787 (N. Maazi)

(C) 2020 Authors. This is an open access publication, which can be used, distributed and reproduced in any medium according to the Creative Commons CC-BY 4.0 License requiring that the original work has been properly cited. 
In the $\mathrm{MC}$ simulation procedure, the random choice is used two times, i.e., in the lattice site to be reoriented and the near neighboring site orientation selections. The strong random nature of the basic MC method is undesirable and may seriously affect the simulation results (Ono et al., 1999; Yu \& Esche, 2003b). A research group (Song \& Liu, 1998; Song et al., 1998; Mason et al., 2015) suggested that there is no physical meaning to randomly selecting lattice sites for reorientation. So a modified MC model is used in the present work. The random choice is only used for the near neighboring site orientation selection. The various selections of one lattice site within every mcs observed in the standard $\mathrm{MC}$ technique are eliminated in the proposed algorithm. The present paper aims to study the influence of the way to choose lattice sites for reorientation on grain growth simulation of a polycrystalline material. The modified MC model's performance is compared to that of a standard MC model by analyzing the microstructures resulting from grain growth simulations using the two models.

\section{Standard MC algorithm of grain growth simulation}

The MC simulation process starts with the definition and initialization of the simulation lattice. The polycrystalline microstructure is mapped on a hexagonal lattice, where each lattice site is assigned a number q that corresponds to the grain orientation at that lattice site (Anderson et al., 1984). All sites within a grain have the same orientation, and GBs are represented by interface lines between neighboring sites of different orientations. In the simulation procedure, a grain site $j\left(q_{j}\right)$ and a near neighboring site $i\left(q_{i}\right)$ are selected randomly. The reorientation attempt of the site $j$ to the site $i\left(q_{j} \rightarrow q_{i}\right)$ is done with the minimization of the energy change $\Delta E$ :

$$
\Delta E=E_{i}-E_{j}
$$

The sites energies $E_{i}$ and $E_{j}$ are computed using the following equation:

$$
E_{k}=\sum_{m=1}^{6} J_{k m}\left(1-\delta\left(q_{k}, q_{m}\right)\right)
$$

where $\delta$ is the Kronecker delta function with $\delta\left(q_{k}, q_{m}\right)=1$ if $q_{k}=q_{m}$ and 0 otherwise, and $J_{k m}$ a positive constant that represents the GB $[\mathrm{km}]$ energy.

The attempted reorientation is accepted if $\Delta E \leq 0$, else, a random number, $r$, between 0 and 1 is generated. The attempted reorientation is accepted if $r \leq P$. $P$ is the transition probability:

$$
P=\left\{\begin{array}{ll}
1 & (\Delta E \leq 0) \\
e^{-\frac{\Delta E}{k T}} & (\Delta E>0)
\end{array}\right\}
$$

where $k$ is the Boltzmann's constant, and $T$ the simulation temperature (not the physical temperature).

Time in the MC Potts model is measured in units of Monte Carlo step (mcs). The number $N$ of reorientation attempts of all matrix sites is defined as one mes.

\section{Results and discussion}

The simulation was started up by using a real microstructure of an electrical steel sheet Fe-3\%Si $\left(1000 \times 1000 \mu \mathrm{m}^{2}\right)$ characterized by Electron Back Scattering Diffraction (EBSD) (Fig. 1). This microstructure corresponds to a hexagonal grid of $N=500 \times 578$ lattice points with an average radius $\bar{R}_{0}=5.33 \mu \mathrm{m}$. The distance between two neighboring lattice points is $\Delta=2 \mu \mathrm{m}$. From EBSD measurements, every lattice point orientation is characterized by the Euler angles $\left(\varphi_{1}, \varphi, \varphi_{2}\right)$. The microstructure is constructed according to the following definition, two lattice points belong to the same grain if the misorientation angle between them is less or equal to $2^{\circ}$, in the contrary case, an interface line of grain boundary separates the two points. Orientation Imaging Microscopy (OIM $\left.{ }^{\mathrm{TM}}\right)$ characterizes the microstructure and the microtexture (grain size distribution, grain boundary type, texture, individual grain orientation, and ODF). Figure 2 shows the ODF's corresponding to the previews microstructure, which have been calculated using the OIMTM maps.

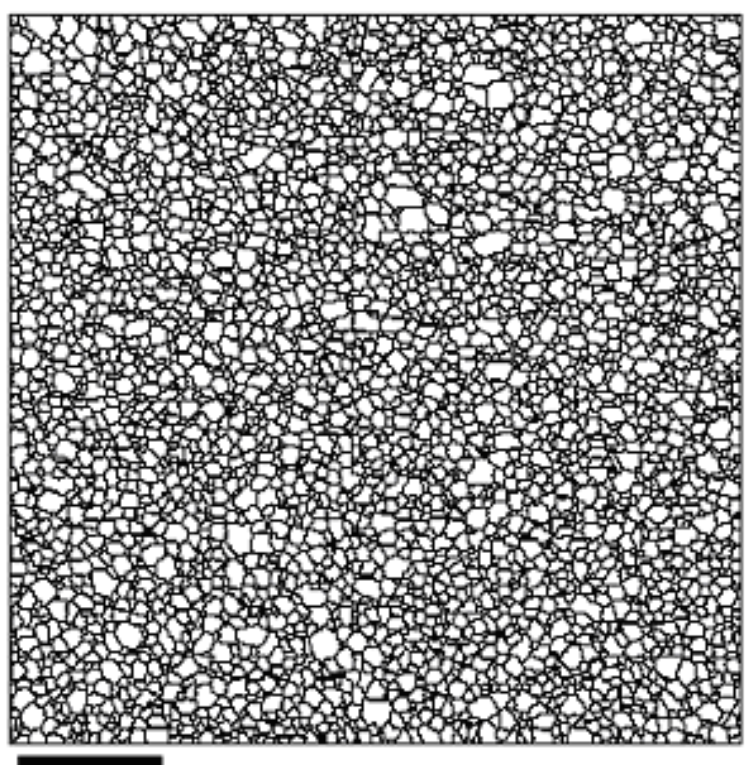

$200.0 \mu \mathrm{m}=100$ steps

Fig. 1. Experimental microstructure characterized by EBSD 


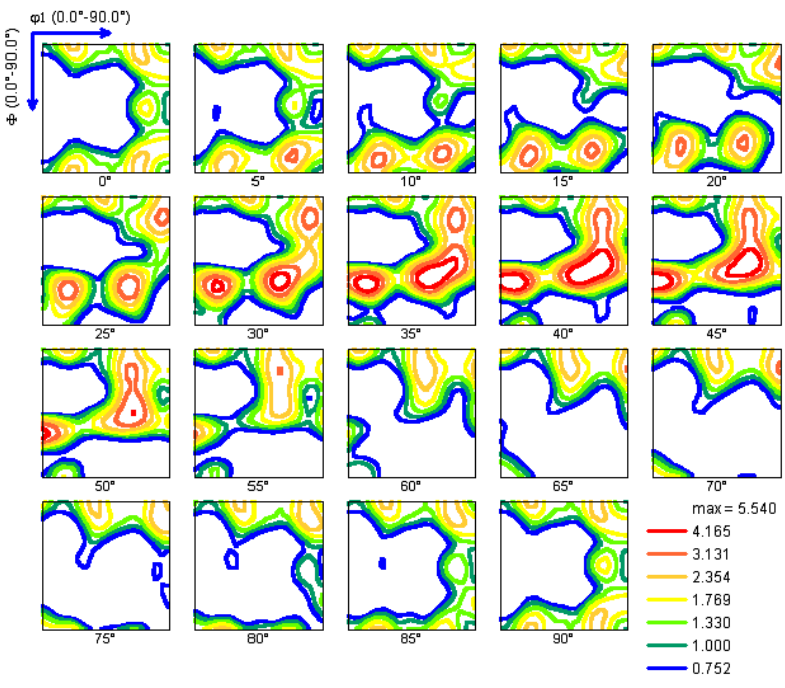

Fig. 2. Constant angle $\varphi_{2}$ sections of the ODF's for the experimental microstructure

\subsection{Modification of the MC algorithm}

Despite its successful results in simulating grain growth behavior, one can make two remarks regarding the basic $\mathrm{MC}$ algorithm of grain growth simulation. First, the MC method is very time-consuming (Bortz et al., 1975; Song et al., 1998). Many enhancements have been made to speed up the basic MC algorithm (Radhakrishnan \& Zacharia, 1995; Holm \& Battaile, 2001; Yu \& Esche, 2003a; Maazi \& Boulechfar, 2019). Second, during the grain growth simulation procedure, the MC algorithm is repeated in random matrix positions. Therefore, some grains are more favorably grown than others due to the several selections of their sites in every mcs, while the remaining grain sites are rarely attempted. To investigate this issue, we ran two series of simulations with the classical MC method ( $\mathrm{MC} 1$ and $\mathrm{MC} 2$ ). Figure 3 represents the microstructure evolution at $t=400 \mathrm{mcs}$ for MC1 and MC2, respectively. It shows a clear difference in the local distribution of grain sizes and shapes (for example, the two zones in the blue and red circles, respectively). The classical MC method permits the evaluation of global behavior under the shape of mean values. The morphological aspect of grain growth simulation is linked to the matrix mean radius and so this can hide the effect of the matrix heterogeneity (Wejrzanowski \& Kurzydlowski, 2005). The grain growth is controlled by the local evolution of grain boundaries around the growing grain and not by the mean evolution of the totality of the matrix (Maazi \& Rouag, 2002).

In the present work, the reoriented lattice sites $j\left(q_{j}\right)$ are respectively chosen one by one following their positions in the matrix from 1 to $\mathrm{N}$ for every mcs, i.e., the first site will be chosen firstly, the second one secondly, and so on. a)

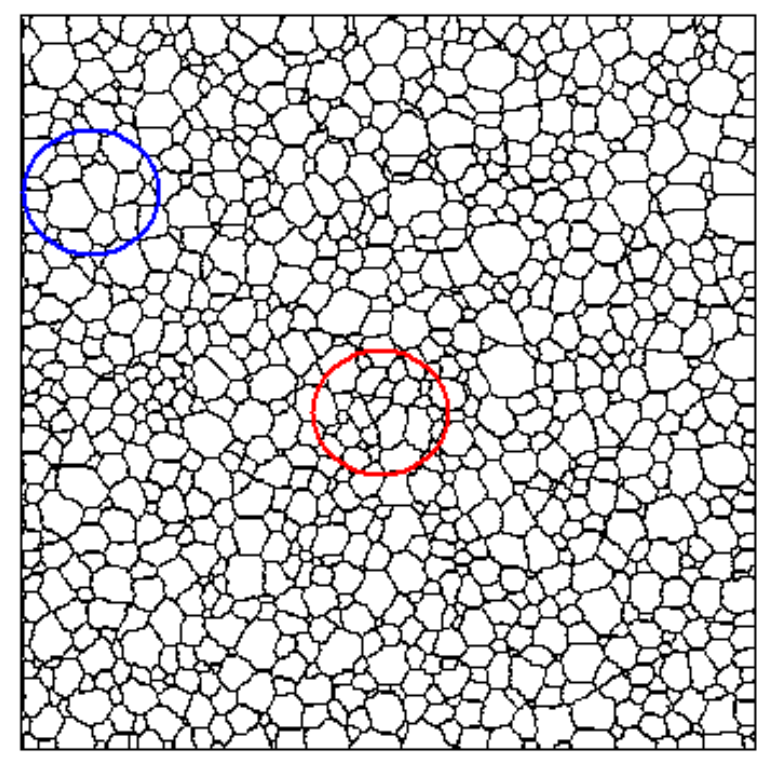

b)

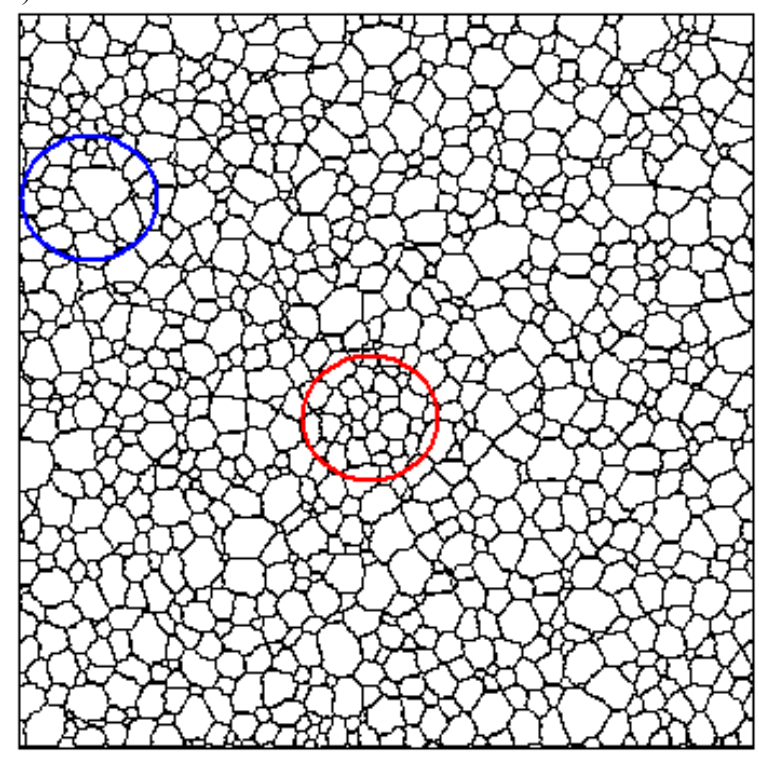

Fig. 3. Microstructure evolution using the classical MC at $t=400$ mcs: a) MC1, b) MC2

Once the reoriented site $j\left(q_{j}\right)$ is chosen, a near neighboring site $i\left(q_{i}\right)$ is randomly chosen for the reorientation test $\left(q_{j} \rightarrow q_{i}\right)$. This modification ensures that the $\mathrm{N}$ matrix sites will be attempted for reorientation exactly once in each mcs, i.e., with the same probability. So reorientation is accepted for different temperatures, with the same probability:

$$
P=\left\{\begin{array}{ll}
1 & (\Delta E \leq 0) \\
0 & (\Delta E>0)
\end{array}\right\}
$$

The real-time and grain growth temperature $T$ can be introduced directly in the simulation procedure using the equation (8) or (9). 


\subsection{Global features of the grain growth process}

In order to test the modified model, a simulation was made for the case of isotropic grain boundaries (All energies and mobilities were set to unity). The microstructure's temporal evolution obtained by the modified model is displayed in Figure $4(t=0-400 \mathrm{mcs})$.

a)

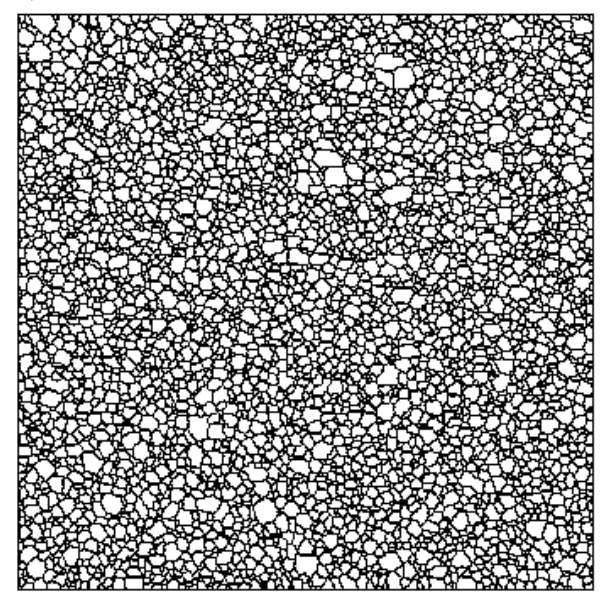

c)

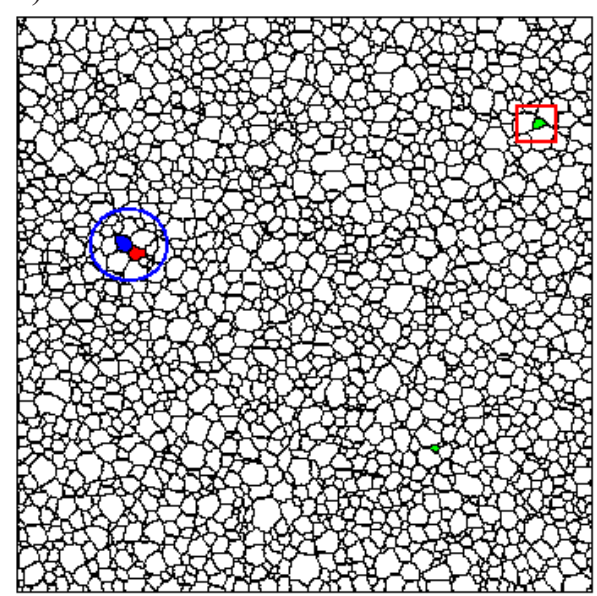

e)

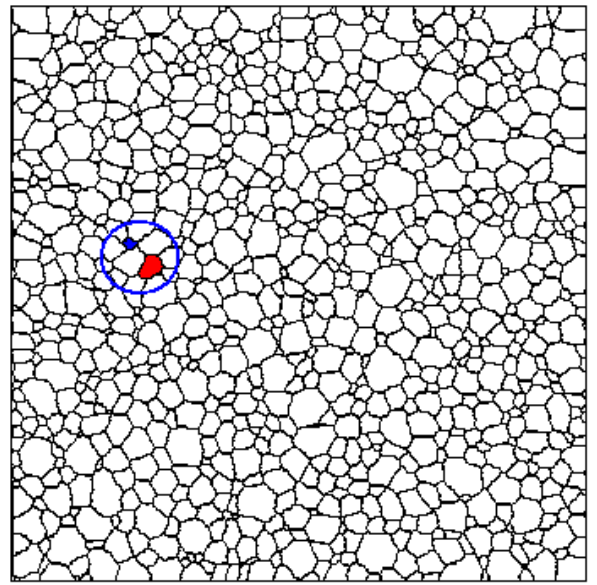

At different stages of the simulation, normal grain growth is observed. The morphological aspect of the grain growth can be related to neighborhood behaviors. One observes that $n$-sided grains grow if $n>6$ and shrink if $n<6$. This behavior is in good agreement with the von Neumann-Mullins law (Neumann, 1952; Mullins, 1956).

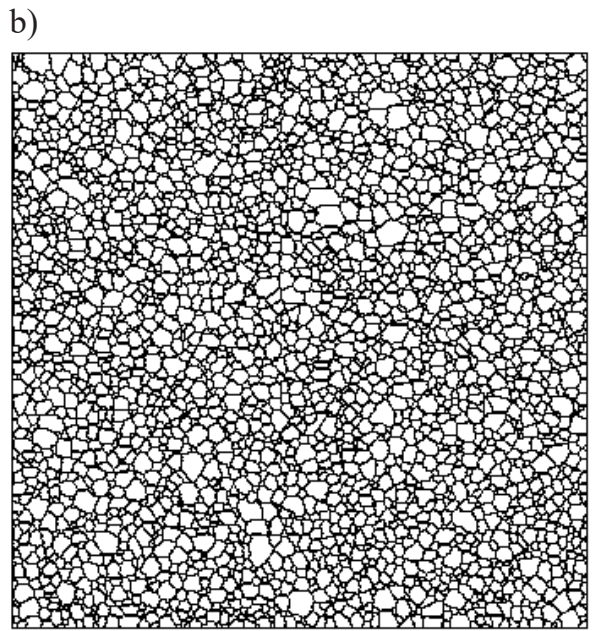

d)

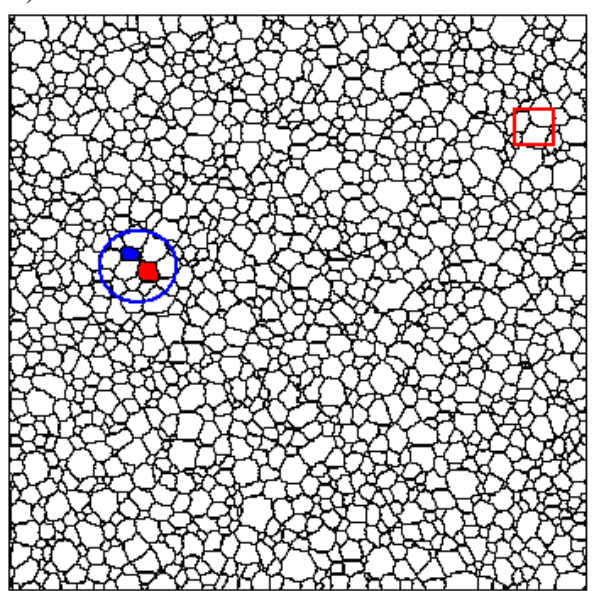

f)

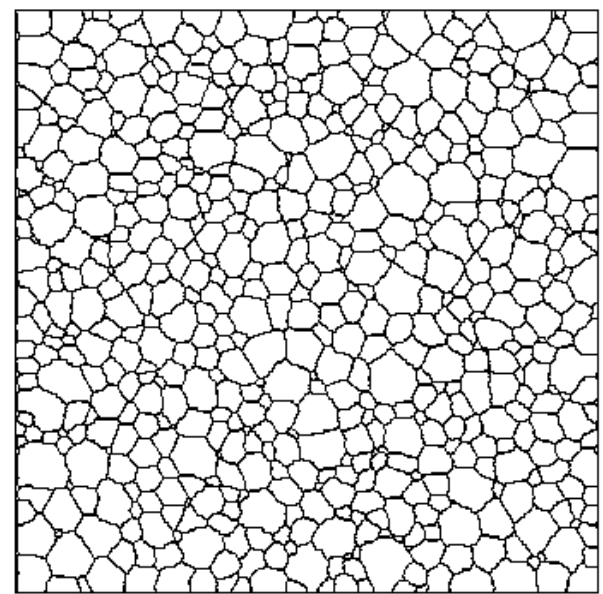

Fig. 4. Microstructure evolution using the modified $\mathrm{MC}$ technique for different mcs: a) $t=0 \mathrm{mcs}$, b) $t=40 \mathrm{mcs}, \mathrm{c}) t=80 \mathrm{mcs}$, d) $t=120 \mathrm{mcs}$, e) $t=240 \mathrm{mcs}, \mathrm{f}) t=400 \mathrm{mcs}$ 
During the growth process, the grain structure undergoes topological changes. In the case of the 2D grain growth, two processes express elementary topological transformations of the microstructure. The first is neighbor switching and the second disappearance of a three-sided grain, which are respectively called the $\mathrm{T} 1$ and the T2 processes (Atkinson, 1988). A number of $\mathrm{T} 1$ and $\mathrm{T} 2$ processes were observed in the present simulation. One example of the topological changes T1 and $\mathrm{T} 2$ is shown in Figure 4c, d, and e, where one uses the circle for T1 (Figs. 4c, d, and e) and the square for T2 (Figs. 4c and d), respectively.

\subsection{Comparison of the modified and standard MC methods}

The modified MC model's performance is compared to that of a standard MC model by analyzing the microstructures resulting from grain growth simulations. The kinetics of normal grain growth is governed by increasing the average grain size $\bar{R}$ according to the following equation (Hillert, 1965):

$$
\bar{R}^{2}-\bar{R}_{0}^{2}=\alpha t
$$

where $\alpha$ is constant, $\bar{R}_{0}$ the initial average grain size of the microstructure, and $t$ the time.

Figure 5 illustrates the time dependence of the mean grain radius $\bar{R}$ for the classical MC and the modified model. It is best fitted by the generalized form in relation (5) for the two cases. To evaluate method efficiency, a parameter e that represents grain growth speed, i.e., the average increasing grain size per mcs, is used. By linear fitting of the data in Figure 5e represents the line slope. The values of e are 0.42 for classical MC and 0.63 for the modified model. It can be seen that the efficiency parameter e of the modified algorithm increases about 2 times compared with that of the classical MC. Then the modified model speeds up the basic MC algorithm of grain growth simulation.

To demonstrate this viewpoint more clearly, Figure 6 represents the microstructure evolution for the classical $(t=400 \mathrm{mcs})$ and the modified $(t=200 \mathrm{mcs})$ models, respectively.

The ODF's corresponding to the previews two microstructures have been calculated using the $\mathrm{OIM}^{\mathrm{TM}}$ maps. Figure 7 indicates that all ODF sections, with the same $\varphi_{2}$, are very similar for the two simulations. For example, from the sections, $\varphi_{2}=45^{\circ}$ the texture consists mainly of a $\gamma$ and $\alpha$ fibers for the two simulations. a)

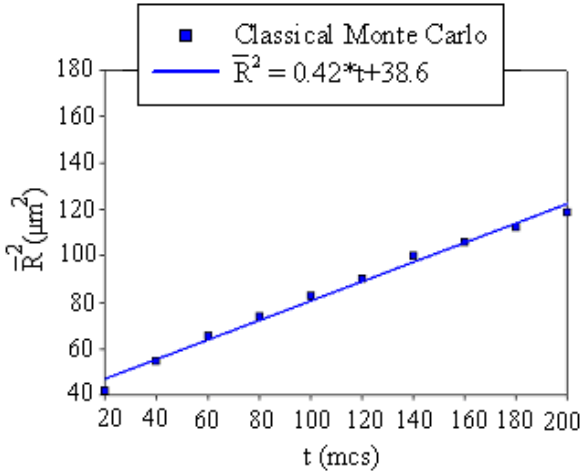

b)

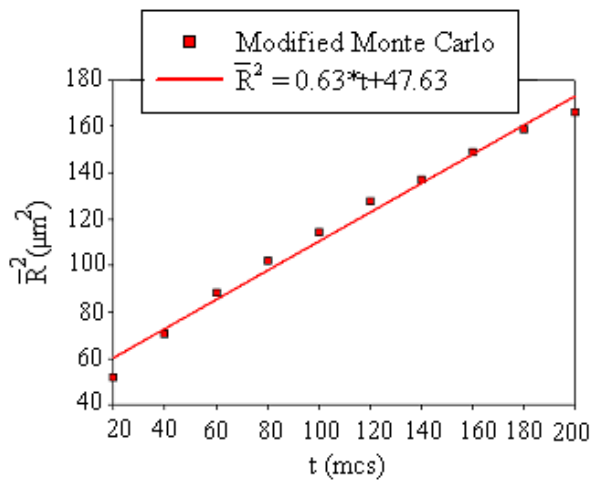

Fig. 5. Square mean-radius variation versus time (mcs) for: a) classical MC, b) the modified model

a)

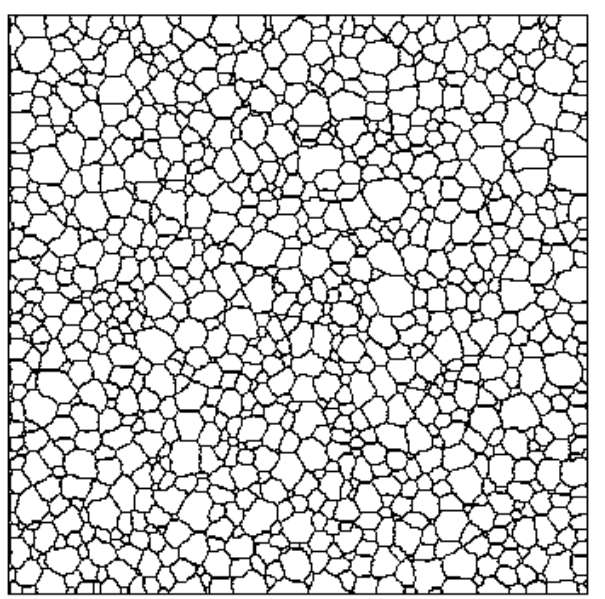

b)

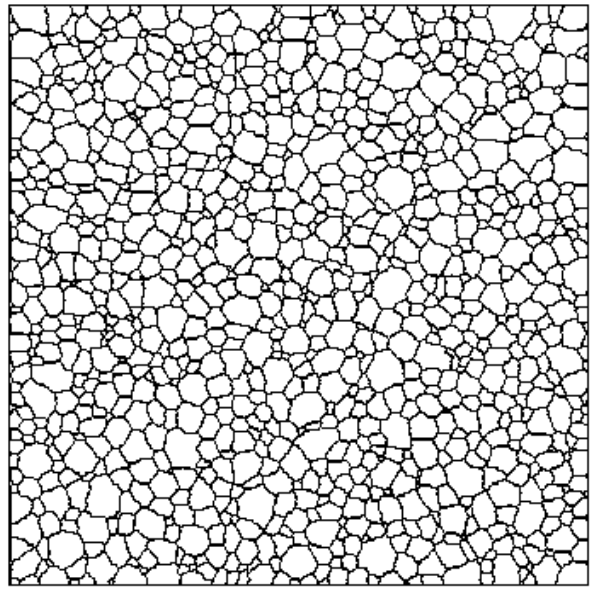

Fig. 6. Microstructural evolution for: a) the classical MC $(t=400 \mathrm{mcs}), \mathrm{b})$ the modified model $(t=200 \mathrm{mcs})$ 


\subsection{The introduction of grain growth temperature and real-time}

Among the shortcomings of the MC technique, one can cite its inability to consider the grain growth phenomenon's physical parameters characteristics. For example, temperature is not directly related to "real" temperature in all basic MC simulations. It can be considered through the transition probability in the reorientation attempts (Eq. 3). So, the temperature influence mechanism on the calculated results in the $\mathrm{MC}$ simulation for grain growth is not well understood. On the other hand, it should be noted that MC models have no real-time of the physical system due to the strong nature of probabilistic transition rules. The correspondence between Monte Carlo steps and real-time is given by (Maazi, 2017):

$$
t(s)=\frac{\sqrt{3} \Delta^{2}}{2 \pi \alpha}\left(\bar{n}-\bar{n}_{0}\right) e^{\frac{Q}{R T}}
$$

where $t(\mathrm{~s})$ is the real-time in seconds, $\bar{n}$ and $\bar{n}=30.8$ are respectively the number of sites contained in the mean matrix area at $t$ and $t=0, T$ the temperature, $R$ the gas constant, and $Q$ the activation energy for grain growth.

From modified MC simulation results, Figure 8 depicts the $\mathrm{MC}$ time dependence of the matrix mean number of sites $\bar{n}$. One obtains by linear fitting a line with an equation:

$$
\bar{n}=1.08 t(\mathrm{mcs})+45.1
$$

Substituting the equation (7) into equation (6) gives the relation between real-time and $t$ (mcs):

$$
t(s)=\frac{\sqrt{3} \Delta^{2}}{2 \pi \alpha}(1.08 t(\mathrm{mcs})+14.3) e^{\frac{Q}{R T}}
$$

and hence:

$$
t(\mathrm{mcs})=\frac{2 \pi \alpha}{1.08 \sqrt{3} \Delta^{2}} e^{-\frac{Q}{R T}} t(s)-13.24
$$

Equation (8) or (9) permits us to introduce the influence of the temperature in the MC simulation instead of using the transition probability (Eq. 3). According to equation (9), the parameters that will be used in the calculation are: $Q=1500 \mathrm{cal} \cdot \mathrm{K} / \mathrm{mol}, \alpha=1$ and $R=2 \mathrm{cal} / \mathrm{mol}$. The influence of the temperature $T$ can be seen from plotting $t(\mathrm{mcs})$ versus the real-time for different temperature values by using the following equation:

$$
t(\mathrm{mcs})=50.40 e^{-\frac{Q}{R T}} t(\min )-13.24
$$



b)

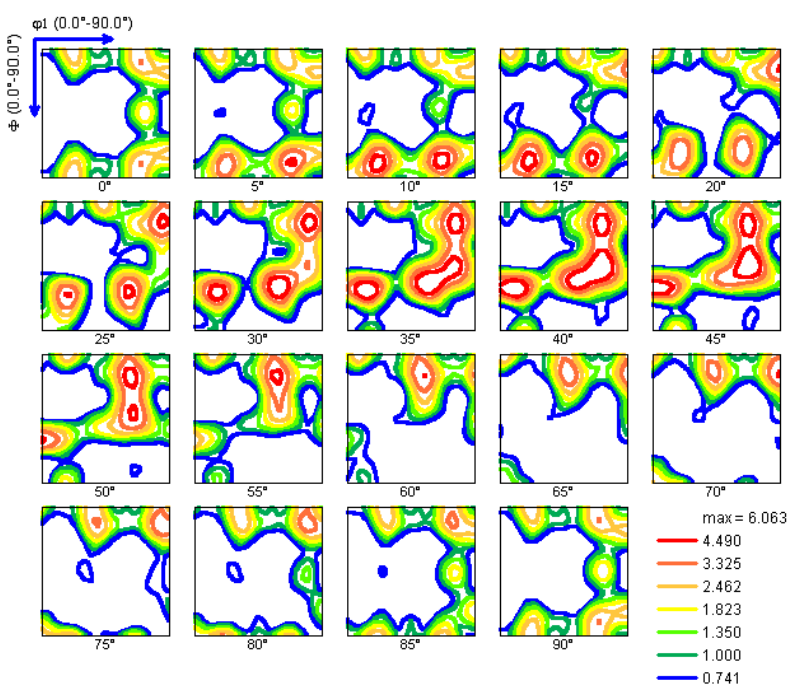

Fig. 7. Constant angle $\varphi_{2}$ sections of the ODF's for: a) classical MC ( $t=400 \mathrm{mcs}), \mathrm{b})$ the modified model $(t=200 \mathrm{mcs})$

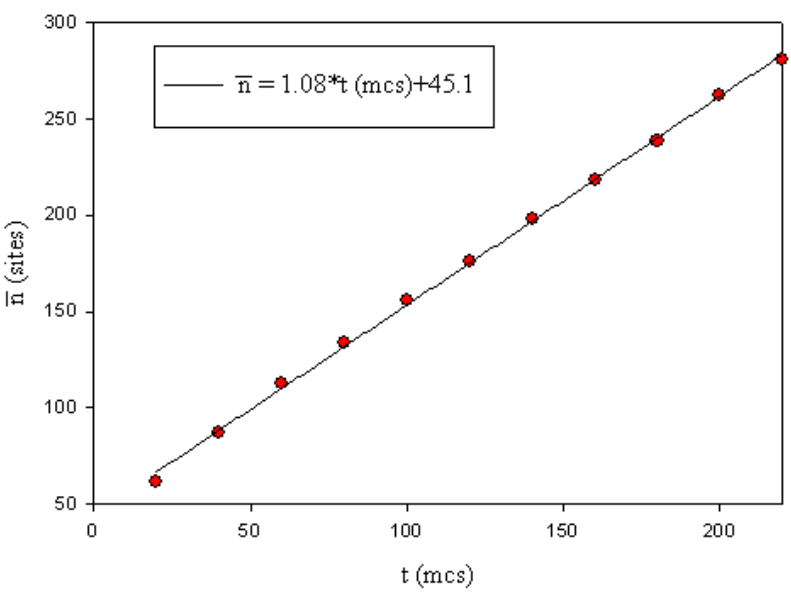

Fig. 8. Variation of mean sites number $\bar{n}$ versus simulated time $t(\mathrm{mcs})$ 
At constant real-time $t$ (min), $t$ (mcs) increases when $T$ increases, as shown in Figure 9. For example, Figure 10 shows the evolution of the microstructure after $10 \mathrm{~min}$ by using the modified $\mathrm{MC}$ model for different values of the temperature: (a) $100^{\circ} \mathrm{C}$, (b) $200^{\circ} \mathrm{C}$, and (c) $500^{\circ} \mathrm{C}$, respectively. The average matrix grain size $\bar{R}$ is $9.98 \mu \mathrm{m}$ for (a), $11.68 \mu \mathrm{m}$ for (b), and $14.88 \mu \mathrm{m}$ for (c). It is clear that the growth is faster at higher grain growth temperature $\left(T=500^{\circ} \mathrm{C}\right)$. These results are in good agreement with the experimental observations concerning the grain growth under different temperatures.

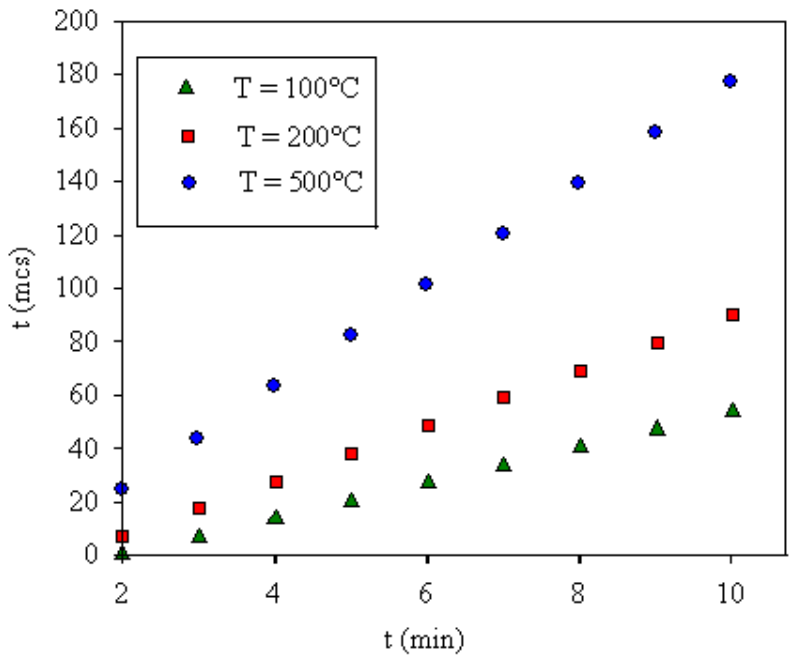

Fig. 9. Variation of the simulated time $t(\mathrm{mcs})$ versus the real time for various temperatures

b)

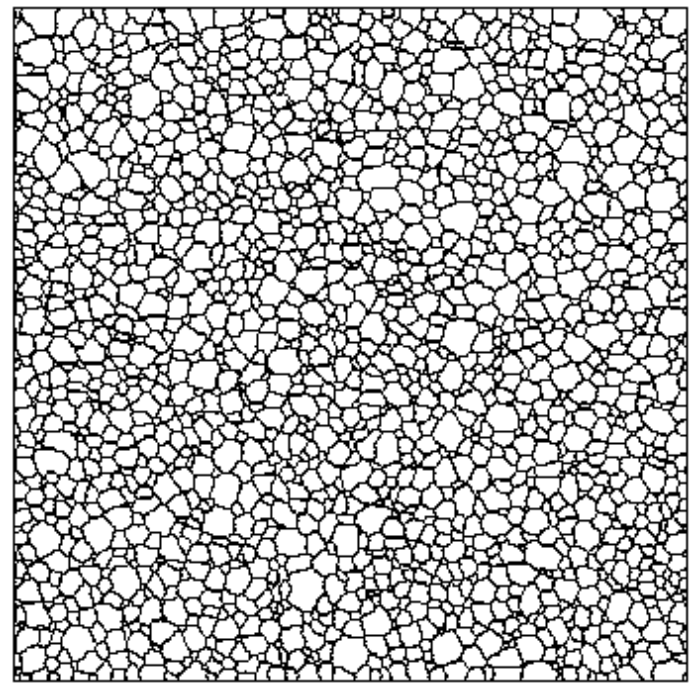

a)

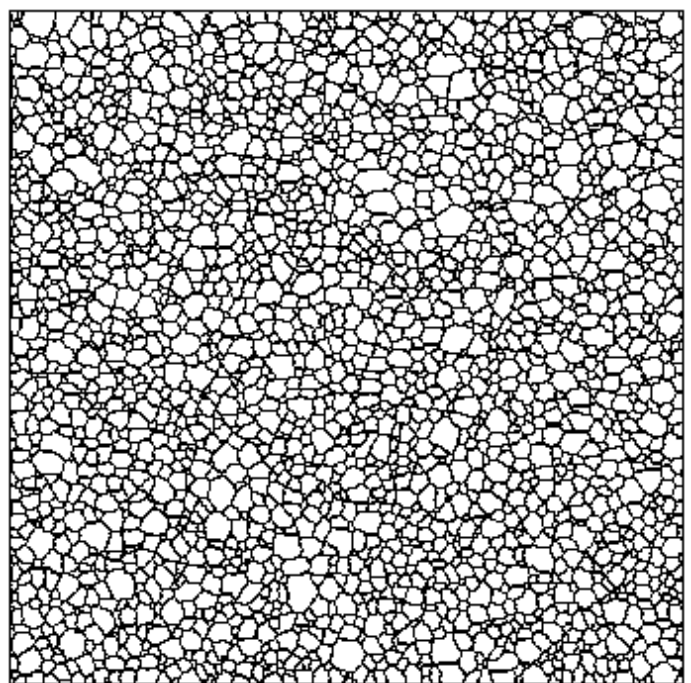

c)

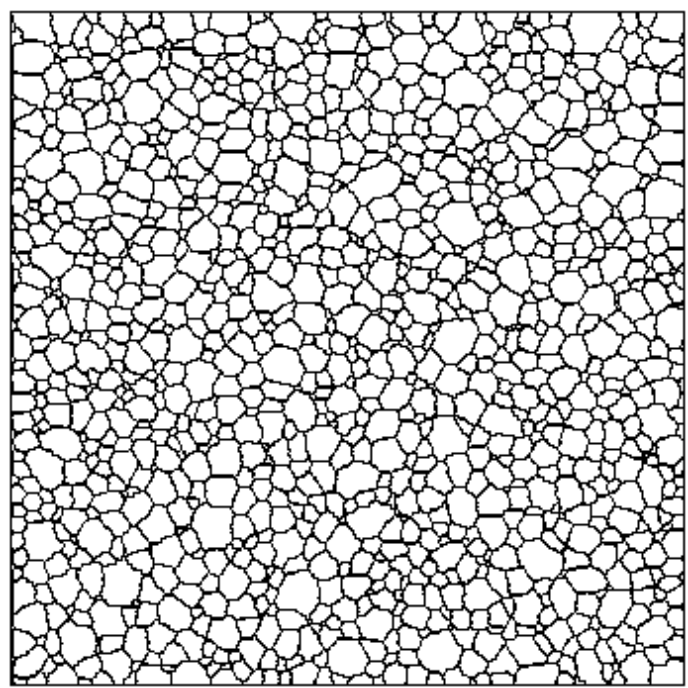

Fig. 10. Simulated grain structure for $t=10 \mathrm{~min}$ at: a) $T=100^{\circ} \mathrm{C}, t=54 \mathrm{mcs}$, b) $T=200^{\circ} \mathrm{C}, t=90 \mathrm{mcs}$, c) $T=500^{\circ} \mathrm{C}, t=178 \mathrm{mcs}$ 


\section{Conclusion}

The strong random nature of the basic MC method is undesirable for grain growth simulation and may seriously affect the simulation results. For example, the random selection of grain sites to be reoriented in the grain growth simulation can lead to the favorable growth of some grains at the others' expense. Effectively, the way to choose lattice sites for reorientation controls the grain growth process. In the modified $\mathrm{MC}$ algorithm, the reoriented grain sites are respectively chosen following their positions in the matrix from 1 to $N$ for every mcs. The random choice is only used for the near neighboring sites orientation selection. This procedure ensures that all lattice sites will be attempted for reorientations exactly once in every Monte Carlo step and no favorable growth of grains at the expense of others. The modified model best reproduces the global features of the grain growth phenomenon. The comparison of the obtained results with and without modifications shows that the modified algorithm significantly increases the calculation speed. The proposed model contributes to overcoming the conventional Monte Carlo computational limitations of grain growth by considering real-time and physical temperature.

\section{References}

Anderson, M.P., Srolovitz, D.J., Grest, G.S., \& Sahni, P.S. (1984). Computer simulation of grain growth - I. Kinetics. Acta Metallurgica, 32(5), 783-791.

Atkinson, H.V. (1988). Overview no. 65: Theories of normal grain growth in pure single phase systems. Acta Metallurgica, 36(3) 469-491.

Bortz, A.B., Kalos, M.H., \& Lebowitz, J.L. (1975). A new algorithm for Monte Carlo simulation of Ising spin systems. Journal of Computational Physics, 17(1), 10-18.

Geiger, J., Roósz, A., \& Barkóczy, P. (2001). Simulation of grain coarsening in two dimensions by cellular-automaton. Acta Materialia, 49(4), 623-629.

Hillert, M. (1965). On the theory of normal and abnormal grain growth. Acta Metallurgica, 13(3), 227-238.

Holm, E.A., \& Battaile, C.C. (2001). The computer simulation of microstructural evolution. JOM. The Journal of The Minerals, Metals \& Materials Society (TMS), 53(9), 20-23.

Holm, E.A., Glazier, J.A., Srolovitz, D.J., \& Grest, G.S. (1991). Effects of lattice anisotropy and temperature on domain growth in the two-dimensional Potts model. Physical Review A, 43(6), 2662-2668.

Kawasaki, K., Nagai, T., \& Nakashima, K. (1989). Vertex models for two-dimensional grain growth. Philosophical Magazine $B, 60(3), 399-421$.

Kim, H.S. (2010). Von Neumann-Mullins equation in the Potts model of two-dimensional grain growth. Computational Materials Science, 50(2), 600-606.

Maazi, N. (2017). Conversion of Monte Carlo steps to real time for grain growth simulation. Advances in Mathematical Physics, 2017, 1-8.

Maazi, N., \& Boulechfar, R. (2019). A modified grain growth Monte Carlo algorithm for increased calculation speed in the presence of Zener drag effect. Materials Science and Engineering: B. 242, 52-62.

Maazi, N., \& Rouag, N. (2002). Consideration of Zener drag effect by introducing a limiting radius for neighbourhood in grain growth simulation. Journal of Crystal Growth, 243(2), 361-369.

Mason, J.K., Lind, J., Li, S.F., Reed, B.W., \& Kumar, M. (2015). Kinetics and anisotropy of the Monte Carlo model of grain growth. Acta Materialia, 82, 155-166.

Messina, R., Soucail, M., \& Kubin, L. (2001). Monte Carlo simulation of abnormal grain growth in two dimensions. Materials Science and Engineering: A, 308(1-2), 258-267.

Mullins, W.W. (1956). Two-Dimensional Motion of Idealized Grain Boundaries. Journal of Applied Physics, 27(8), 900-904.

Neumann, J. von (1952). Discussion - shape of metal grains. In Metal interfaces a seminar on metal interfaces held during the Thirty-third National Metal Congress and Exposition, Detroit, October 13 to 19, 1951 (pp. 108-110). American Society for Metals.

Ono, N., Kimura, K., \& Watanabe, T. (1999). Monte Carlo simulation of grain growth with the full spectra of grain orientation and grain boundary energy. Acta Materialia, 47(3), 1007-1017.

Phaneesh, K.R., Bhat, A., Mukherjee, P., \& Kashyap, K.T. (2012). On the Zener limit of grain growth through 2D Monte Carlo simulation. Computational Materials Science, 58, 188-191.

Radhakrishnan, B., \& Zacharia, T. (1995). Simulation of curvature-driven grain growth by using a modified Monte Carlo algorithm. Metallurgical and Materials Transactions A, 26(1), 167-180.

Raghavan, S., \& Sahay, S. (2007). Modeling the grain growth kinetics by cellular automaton. Materials Science and Engineering: A, 445-446, 203-209.

Song, X., \& Liu, G. (1998). A simple and efficient three-dimensional Monte Carlo simulation of grain growth. Scripta Materialia, 38(11), 1691-1696.

Song, X., Liu, G., \& He, Y. (1998). Modified Monte Carlo method for grain growth simulation. Progress in Natural Science, 8, 92-97. 
Srolovitz, D.J., Grest, G.S., \& Anderson, M.P. (1986). Computer simulation of recrystallization - I. Homogeneous nucleation and growth. Acta Metallurgica, 34(9), 1833-1845.

Suwa, Y., Saito, Y., \& Onodera, H. (2006). Phase field simulation of grain growth in three dimensional system containing finely dispersed second-phase particles. Scripta Materialia, 55(4), 407-410.

Wang, Y.U. (2006). Computer modeling and simulation of solid-state sintering: A phase field approach. Acta Materialia, 54(4), 953-961.

Wejrzanowski, T., \& Kurzydlowski, K.J. (2005). Modelling of the influence of the grain size distribution on the grain growth in nanocrystals. Solid State Phenomena, 101-102, 315-318.

Weygand, D., Bréchet, Y., \& Lépinoux, J. (2000). Inhibition of grain growth by particle distribution: effect of spatial heterogeneities and of particle strength dispersion. Materials Science and Engineering: A, 292(1), 34-39.

Yu, Q., \& Esche, S.K. (2003a). A Monte Carlo algorithm for single phase normal grain growth with improved accuracy and efficiency. Computational Materials Science, 27(3), 259-270.

Yu, Q., \& Esche, S.K. (2003b). A new perspective on the normal grain growth exponent obtained in two-dimensional Monte Carlo simulations. Modelling and Simulation in Materials Science and Engineering, 11(6), 859-861. 
